\title{
A FUNCTION SPACE INTEGRAL FOR A BANACH SPACE OF FUNCTIONALS ON WIENER SPACE
}

\author{
G. W. JOHNSON AND D. L. SKOUG
}

\begin{abstract}
In an earlier paper the authors established the existence of Cameron and Storvick's function space integral $J_{a}(F)$ for a class of finite-dimensional functionals $F$. Here we consider a space $A$ of not necessarily finite-dimensional functionals generated by the earlier functionals. We show that $A$ is a Banach space and recognize $A$ as the direct sum of more familiar Banach spaces. We also show that the function space integral $J_{a}^{\text {an }}(F)$ exists for $F \in A$. In contrast we give an example of an $F_{0} \in A$ such that $J_{q}^{\text {seq }}\left(F_{0}\right)$ does not exist.
\end{abstract}

0. Introduction. Cameron and Storvick introduced the "integral" of the title in [2]. Insofar as possible, we adopt the definitions and notation of [2]. "Integrals" closely related to [2] have been studied by others including Babbitt, Beekman and Kallman, Cameron, Feldman, and Nelson. The initial motivation for the study of these integrals is found in the work of the physicist Feynman. For references see [2] and [1].

Let $\left\{\alpha_{1}, \alpha_{2}, \cdots, \alpha_{n}, \cdots\right\}$ be a fixed complete orthonormal set of real functions of bounded variation on $[a, b]$ with $\alpha_{1}(t) \equiv(b-a)^{-1 / 2}$ the normalized constant function. Given a finite subset $\left\{\alpha_{i_{1}}, \cdots, \alpha_{i_{m}}\right\}$ and a complex-valued function $f \in L_{1}\left(R^{m}\right)$, let a functional $F$ on Wiener space $C_{0}[a, b]$ be defined by

$$
F(x)=f\left(\int_{a}^{b} \alpha_{i_{1}}(t) d x(t), \cdots, \int_{a}^{b} \alpha_{i_{m}}(t) d x(t)\right) .
$$

(It will be convenient to denote the set of positive integers $\left\{i_{1}, \cdots, i_{m}\right\}$ by $\{(i, 1),(i, 2), \cdots,(i, m)\}$.) Under the above assumptions, the present authors established [3] the existence of the function space integral $J_{q}(F)$ for all real $q \neq 0$. (For definition of $J_{q}(F)$ see [2, pp. 533-534].) Here we assume in addition that $f \in L_{\infty}\left(R^{m}\right)$. Given such an $f$, let

$$
\|f\| \equiv\|f\|_{\infty}+(m / 2 \pi e)^{m / 2}\|f\|_{1} .
$$

Presented to the Society, January 18, 1974; received by the editors September 20, 1972 and, in revised form, June 4, 1973.

AMS (MOS) subject classifications (1970). Primary 28A40.

Key words and phrases. Wiener integral, operator valued function space integral, Feynman integral, Banach space.

(C) American Mathematical Society 1974 
(The reason for the constant in (0.2) will become clear in $\$ 2$ below.) The space $A$ will consist of functionals on $C_{0}[a, b]$ of the form $F=\sum_{1}^{\infty} F_{j}$ where each $F_{j}$ has the form $(0.1)$ and $\sum_{1}^{\infty}\left\|f_{j}\right\|<\infty$. We will show that there is a canonical way of writing the elements of $A$ which is essentially unique and then will define a norm $N$ on $A$ in terms of this representation. We then get that $A$ is isometrically isomorphic to a countable direct sum of spaces each of which is essentially $\left(\left(L_{1} \cap L_{\infty}\right)\left(R^{m}\right),\|\cdot\|_{\infty}+\|\cdot\|_{1}\right)$ for some integer $m$. It will follow that $(A, N)$ is a Banach space.

Finally we show that $F \in A$ implies the existence of $J_{q}^{\text {an }}(F)$ but not $J_{q}^{\text {seq }}(F)$. (For definitions, see [2, pp. 533-534].) The counterexample shows rather strikingly how pathological the sequential function space integral can be; the $F_{0} \in A$ such that $J_{q}^{\text {seq }}\left(F_{0}\right)$ fails to exist is equivalent to 0 .

1. The canonical representation. Let $A_{0}$ be the set of all functionals on $C_{0}[a, b]$ of the form $(0.1)$ where $\|f\|<\infty$. Let $\left\{F_{j}\right\}$ be a sequence from $A_{0}$ such that $\sum_{1}^{\infty}\left\|f_{j}\right\|<\infty$. Given $x \in C_{0}[a, b]$, let

$$
F(x) \equiv \sum_{1}^{\infty} F_{j}(x)
$$

Now for every $\lambda>0,\left\|f_{j}\right\|_{\infty}$ is an essential bound for $\left|F_{j}\left(\lambda^{-1 / 2} x\right)\right|$. This is quite easily seen using a fundamental Wiener integration formula. (Since the proof of this fact is essentially carried out as part of the proof of Theorem 2.2 below we will omit the proof here.) It follows that for every $\lambda>0$, the series $\sum_{1}^{\infty} F_{j}\left(\lambda^{-1 / 2} x\right)$ is absolutely convergent for a.e. $x$. We let $A$ denote the collection of all functionals $F$ arising as above from a sequence $\left\{F_{j}\right\}$ from $A_{0}$.

Given $F=\sum_{1}^{\infty} F_{j}$ in $A$, one may add together all the $F_{j}$ 's in the sum which are based on exactly the same set of $\alpha$ 's and get a new series

$$
\sum_{1}^{\infty} G_{j}(x)=\sum_{1}^{\infty} g_{j}\left(\int_{a}^{b} \alpha_{j, 1}(t) d x(t), \cdots, \int_{a}^{b} \alpha_{j, m_{j}}(t) d x(t)\right)
$$

where $\sum_{1}^{\infty}\left\|g_{j}\right\| \leqq \sum_{1}^{\infty}\left\|f_{j}\right\|<\infty$ and where

$$
\left\{(i, 1), \cdots,\left(i, m_{i}\right)\right\} \neq\left\{(j, 1), \cdots,\left(j, m_{j}\right)\right\} \text { for } i \neq j .
$$

The new series still sums to $F$ because of the absolute convergence. We show below that the representation (1.2) for $F$ under condition (1.3) is unique up to the order of the sum and changes in $g_{j}$ on a set of measure zero in $R^{m_{j}}$. This result is a key step in the development. Once this is done we unambiguously define $N$ on $A$ by

$$
N(F) \equiv \sum_{j}\left\|g_{j}\right\|
$$

and the following theorem is immediate. 
THEOREM 1.1. The space $A$ is a normed linear space under the norm $N$.

Much of the present section concentrates on proving the uniqueness of the representation (1.2) subject to (1.3). Part of the argument takes place in Euclidean space. We use $\mu$ (somewhat ambiguously) to refer to Lebesgue measure on $R^{n}$ for any $n$. The first lemma is an easy consequence of Fubini's theorem.

LEMMA 1.1. Let $B \subseteq R^{m+n}$ satisfy $\mu\left(B^{c}\right)<\infty$; then for almost every (a.e.) $v \in R^{n}, \mu\left(B^{c}(v)\right)<\infty$ where $B(v)$ denotes the $v$-section of $B$.

We need to consider sets which are the Cartesian product of some copies of $R$ with a set $B$ satisfying $\mu\left(B^{c}\right)<\infty$. The next lemma along with Lemma 1.1 tells us that a.e. section of such a set is again such a set.

Let $\tau \subseteq P \equiv\{1,2, \cdots, n\}$ and let $\rho=P \backslash \tau$. Our sets will have the form $B_{\tau} \times R_{\rho}$ where $\mu\left(B_{\tau}^{c}\right)<\infty$. (For example, let $n=4, \tau=\{1,3\}$; then $B_{\tau} \subseteq R_{1} \times$ $R_{3}$ and $B_{\tau} \times R_{\rho}=\left\{\left(v_{1}, v_{2}, v_{3}, v_{4}\right):\left(v_{1}, v_{3}\right) \in B_{\tau}\right\}$.) We allow the possibilities $\tau=\varnothing, \tau=P$.

Lemma 1.2. Let $\alpha \subseteq P$ be such that $\tau \mid \alpha \neq \varnothing$. Then for $v_{\alpha} \in R_{\alpha}$,

$$
\left(B_{\tau} \times R_{\rho}\right)\left(v_{\alpha}\right)=B_{\tau}\left(v_{\tau \cap \alpha}\right) \times R_{\rho \backslash \alpha}
$$

where $v_{\tau \cap \alpha}$ denotes the projection of $v_{\alpha}$ into $R_{\tau \cap \alpha}$.

We need a lemma insuring that the intersection of a finite number of sets as in Lemma 1.2 has infinite measure.

LemMa 1.3. Let $n$ be a positive integer. For $i=1,2, \cdots, m$, let $\tau_{i} \subseteq P=\{1,2, \cdots, n\}$, let $\rho_{i}=P \backslash \tau_{i}$, let $A_{\tau_{i}} \subseteq R_{r_{i}}$ with $\mu\left(A_{\tau_{i}}^{c}\right)<\infty$, and let $E_{i}=A_{\tau_{i}} \times R_{\rho_{i}}$. Then $E \equiv \bigcap_{i=1}^{m} E_{i}$ has infinite measure.

Proof. The result is clear for $m=1$. Now assume the result for $m$ sets and any dimension and examine the case of $m+1$ sets and any dimension (say $n$ ). If $\tau_{i}=\tau_{j}$ for some $i \neq j$, then $E_{i} \cap E_{j}$ can be written as one set of the type under consideration and we may apply the induction assumption. So we may suppose that $i \neq j$ implies $\tau_{i} \neq \tau_{j}$. Also if any $\tau_{i}$ is empty, then $E_{i}=R^{n}$ and the intersection may again be reduced to $m$ sets. Now choose $i_{0} \in\{1,2, \cdots, m+1\}$ such that $\tau_{i_{0}}$ has a minimal number of elements. We may suppose $i_{0}=m+1$. Then for $1 \leqq i \leqq m, \tau_{i} \mid \tau_{m+1} \neq \varnothing$. Let $v_{\tau_{m+1}} \in A_{\tau_{m+1}}$. We will show that $E\left(v_{\tau_{m+1}}\right)$ has infinite measure for a.e. $v_{\tau_{m+1}}$ in $A_{\tau_{m+1}}$. But

$$
E\left(v_{\tau_{m+1}}\right)=\bigcap_{i=1}^{m+1} E_{i}\left(v_{\tau_{m+1}}\right)=\left[\bigcap_{i=1}^{m} E_{i}\left(v_{\tau_{m+1}}\right)\right] \cap R_{\rho_{m+1}} .
$$

The result follows from Lemmas $1.1,1.2$, and the induction hypothesis. 
THEOREM 1.2. The representation (1.2) of an $F \in A$ subject to (1.3) is unique.

Proof. It suffices to show that 0 has a unique representation and, for this, it suffices to examine a sum of the form (1.2) where $g_{j} \neq 0$ for all $j$ and show that $G(x) \equiv \sum_{j=1}^{\infty} G_{j}(x)$ is not the zero functional on $C_{0}[a, b]$. For $j=1,2, \cdots$, let $\tau_{j}$ be the set of positive integers $\left\{(j, 1), \cdots,\left(j, m_{j}\right)\right\}$ and let $V_{r_{j}}$ be the vector $\left(v_{j, 1}, \cdots, v_{j, m_{j}}\right)$. We may arrange the sum so that $m_{1} \leqq m_{j}$ for $j=2,3, \cdots$. Since $g_{1} \neq 0$ there exists $\varepsilon>0$ and a set $A_{1} \subseteq R_{\tau_{1}}$ of positive measure such that $\left|g_{1}\left(V_{\tau_{1}}\right)\right| \geqq \varepsilon$ on $A_{1}$. Now $\sum_{1}^{\infty}\left\|g_{j}\right\|_{\infty}<\infty$ and so there exists $K \geqq 2$ such that $\sum_{K+1}^{\infty}\left\|g_{j}\right\|_{\infty}<\varepsilon / 4$. Now $\mu\left(M_{j}\right)=0$ where $M_{j}=\left\{V_{r_{j}} \in R_{r_{j}}:\left|g_{j}\left(V_{r_{j}}\right)\right|>\left\|g_{j}\right\|_{\infty}\right\}$. Hence

$w\left\{x \in C_{0}[a, b]:\left|G_{j}(x)\right|>\left\|g_{j}\right\|_{\infty}\right\}$

$$
\begin{aligned}
& =\int_{C_{0}[a, b]} \chi_{M_{j}}\left(\int_{a}^{b} \alpha_{j, 1}(t) d x(t), \cdots, \int_{a}^{b} \alpha_{j, m_{j}}(t) d x(t)\right) d x \\
& =\left(\frac{1}{2 \pi}\right)^{m_{j} / 2} \int_{-\infty}^{\infty}\left(m_{j}\right) \int_{-\infty}^{\infty} \chi_{M_{j}}\left(v_{j, 1}, \cdots, v_{j, m_{j}}\right) \\
& \quad \cdot \exp \left\{-\frac{1}{2}\left(v_{j, 1}^{2}+\cdots+v_{j, m_{j}}^{2}\right)\right\} d v_{j, 1} \cdots d v_{j, m_{j}},
\end{aligned}
$$

where $w$ denotes Wiener measure. It follows that $w\left\{x \in C_{0}[a, b]\right.$ : $\left.\sum_{K+1}^{\infty}\left|G_{j}(x)\right| \geqq \varepsilon / 4\right\}=0$. The proof will be complete if we show that $w\left(H_{K}\right)>$ 0 where $H_{K} \equiv\left\{x \in C_{0}[a, b]:\left|G_{1}(x)\right|>\varepsilon, \quad\left|G_{2}(x)\right|<\varepsilon / 4 K, \cdots,\left|G_{K}(x)\right|<\right.$ $\varepsilon / 4 K\}$. Let $\tau=\bigcup_{j=1}^{K} \tau_{j}$. It suffices to show that $\mu(L)>0$ where $L \equiv \bigcap_{1}^{K} L_{j}$, $L_{1} \equiv\left\{V_{\tau} \in R_{\tau}:\left|g_{1}\left(V_{\tau_{1}}\right)\right|>\varepsilon\right\}, L_{j} \equiv\left\{V_{\tau} \in R_{\tau}:\left|g_{j}\left(V_{r_{j}}\right)\right|<\varepsilon / 4 K\right\}$ for $j=2, \cdots, K$, since $w\left(H_{K}\right)>0$ will follow from an argument as in (1.5). Note that for $j=2, \cdots, K, L_{j}$ is a set of the type discussed in Lemma 1.3 since $g_{j} \in L_{1}\left(R_{r_{j}}\right)$. Now $\tau_{j} \mid \tau_{1} \neq \varnothing$ for $j=2, \cdots, K$ and to show $\mu(L)>0$, it suffices to show that $\mu\left(L\left(V_{T_{1}}\right)\right)=\infty$ for a.e. $V_{T_{1}} \in A_{1}$. However $L\left(V_{T_{1}}\right)=$ $\bigcap_{j=1}^{K} L_{j}\left(V_{\tau_{1}}\right)=\bigcap_{j=2}^{K} L_{j}\left(V_{\tau_{1}}\right)$ and this set has infinite measure for a.e. $V_{\tau_{1}} \in A_{1}$ by Lemmas $1.1,1.2$, and 1.3 .

Theorem 1.1 now follows with $N$ given by (1.4).

Let $T=\left\{\tau_{1}, \tau_{2}, \cdots\right\}$ be an enumeration of the collection of all finite subsets of the positive integers where $\tau_{j}=\left\{(j, 1), \cdots,\left(j, m_{j}\right)\right\}$. Associate with $\tau_{j}$ the Banach space $B\left(\tau_{j}\right) \equiv\left(\left(L_{1} \cap L_{\infty}\right)\left(R_{r_{j}}\right),\|\cdot\|_{r_{j}}\right)$ where $\|\cdot\|_{r_{j}} \equiv$ $\|\cdot\|_{\infty}+\left(m_{j} / 2 \pi e\right)^{m_{j} / 2}\|\cdot\|_{1}$. Note that $B\left(\tau_{j}\right)$ is equivalent to

$$
\left(\left(L_{1} \cap L_{\infty}\right)\left(R_{\tau_{j}}\right),\|\cdot\|_{\infty}+\|\cdot\|_{1}\right) \text {. }
$$

Then the set $\sum_{j} \oplus B\left(\tau_{j}\right)$ of all sequences $\left\{g_{\tau_{j}}\right\}$ such that $\sum_{j}\left\|g_{\tau_{j}}\right\|_{\tau_{j}}<\infty$ is a Banach space under the norm $\left\|\left\{g_{r_{j}}\right\}\right\|_{B} \equiv \sum_{j}\left\|g_{\tau_{j}}\right\|_{r_{j}}$. The following theorem is now quite clear from (0.2), (1.4), and Theorem 1.2. 
THEOREM 1.3. $(A, N)$ is isometrically isomorphic to $\left(\sum_{j} \oplus B\left(\tau_{j}\right),\|\cdot\|_{B}\right)$.

Proof. The appropriate correspondence sends $\left\{g_{\tau_{j}}\right\}$ into the functional on $C_{0}[a, b]$ carrying $x$ to $\sum_{j=1}^{\infty} g_{\tau_{j}}\left(\int_{a}^{b} \alpha_{j, 1}(t) d x(t), \cdots, \int_{a}^{b} \alpha_{j, m_{j}}(t) d x(t)\right)$.

2. $J_{q}^{\text {an }}(F)$ and $J_{q}^{\text {seq }}(F)$.

Lemma 2.1. Suppose $F$ is given by (0.1) with $f \in L_{1}\left(R^{m}\right)$. Then $I_{\lambda}^{\text {an }}(F)$ and $J_{q}^{\mathrm{an}}(F)$ exist for all $\lambda \in C^{+} \equiv\{\lambda: \operatorname{Re} \lambda>0\}$ and all real $q \neq 0$ and satisfy:

$$
\begin{aligned}
\left\|I_{\lambda}^{\mathrm{an}}(F)\right\| & \leqq(|\lambda| / 2 \pi)^{m / 2}\|f\|_{1} \leqq e^{|\lambda| / 2}(m / 2 \pi e)^{m / 2}\|f\|_{1} \leqq e^{|\lambda| / 2}\|f\|, \\
\left\|J_{q}^{\mathrm{an}}(F)\right\| & \leqq(|q| / 2 \pi)^{m / 2}\|f\|_{1} \leqq e^{|q| / 2}(m / 2 \pi e)^{m / 2}\|f\|_{1} \leqq e^{|q| / 2}\|f\| .
\end{aligned}
$$

Proof. The existence of $I_{\lambda}^{\mathrm{an}}(F)$ and $J_{q}^{\mathrm{an}}(F)$ (in fact $I_{\lambda}(F)$ and $J_{q}(F)$ ) was established in [3, Theorems 1 and 2]. The first inequalities in (2.1) and (2.2) are not explicitly stated in [3] but they follow easily from the expressions for $\left\|I_{\lambda}(F)\right\|$ and $\left\|J_{q}(F)\right\|$ in [3, pp. 421-422] and the fact that for an $L_{1}$-function $h,\|\mathscr{F}(h)\|_{\infty} \leqq\|h\|_{1}$ where $\mathscr{F}$ denotes the Fourier transform. Now

$$
\left(\frac{|\lambda|}{2 \pi}\right)^{m / 2} \leqq e^{|\lambda| / 2} \sup _{\lambda \geqq 0}\left\{e^{-\lambda / 2}\left(\frac{\lambda}{2 \pi}\right)^{m / 2}\right\}=\left(\frac{m}{2 \pi e}\right)^{m / 2} e^{|\lambda| / 2}
$$

and so the second inequalities in (2.1) and (2.2) are verified. The last inequalities are clear by definition of $\|f\|$.

THeOrem 2.1. Let $F \in A$. Then $I_{\lambda}^{\mathrm{an}}(F)$ and $J_{q}^{\mathrm{an}}(F)$ exist for all $\lambda \in C^{+}$ and all real $q \neq 0$ and satisfy

$$
\left\|I_{\lambda}^{\text {an }}(F)\right\| \leqq e^{|\lambda| / 2} N(F) \quad \text { and } \quad\left\|J_{q}^{\text {an }}(F)\right\| \leqq e^{|q| / 2} N(F) .
$$

Remarks. (i) To show that $J_{q}^{\text {an }}(F)$ exists it would suffice to show $\left[2\right.$, p. 534] that $J_{q}^{\text {an }}(F)$ is the weak operator limit of $I_{p-i q}^{\text {an }}(F)$ as $p \rightarrow 0^{+}$; in fact, we show $J_{q}^{\text {an }}(F)$ to be the strong operator limit of $I_{\lambda}^{\text {an }}(F)$ as $\lambda \rightarrow-i q$ in $C^{+}$.

(ii) Combining the explicit formulas in [3] with the work below we actually can give explicit formulas for $J_{q}^{\text {an }}(F), F \in A$. One of the difficulties in studying the Cameron-Storvick theory has been the lack of explicitly computable examples; the present paper should alleviate this problem somewhat.

Proof. Suppose $F \in A$ is given in its canonical representation by (1.2). First we show that $I_{\lambda}^{\mathrm{an}}(F)$ exists for $\lambda \in C^{+}$. Let

$$
L_{\lambda}(F) \equiv \sum_{1}^{\infty} I_{\lambda}^{\text {an }}\left(G_{j}\right) \text {. }
$$


By (2.1), $\sum_{1}^{\infty}\left\|I_{\lambda}^{\text {an }}\left(G_{j}\right)\right\| \leqq e^{|\lambda| / 2} \sum_{1}^{\infty}\left\|g_{j}\right\|=e^{|\lambda| / 2} N(F)$. Hence $L_{\lambda}(F)$ is an operator satisfying $\left\|L_{\lambda}(F)\right\| \leqq e^{|\lambda| / 2} N(F)$. Also the partial sums of (2.4) are seen to converge uniformly to $L_{\lambda}(F)$ on any bounded subset of $C^{+}$ and, since these partial sums are analytic functions of $\lambda, L_{\lambda}(F)$ is analytic in $\lambda$. It remains to show that $L_{\lambda}(F) \psi$ agrees with

$$
\int_{C_{0}[a, b]} F\left(\lambda^{-1 / 2} x+\xi\right) \psi\left(\lambda^{-1 / 2} x(b)+\xi\right) d x \text { for } \lambda>0 .
$$

By Lemma 2.1 and the dominated convergence theorem

$$
\begin{aligned}
\int_{C_{0}[a . b]}\left[\sum_{1}^{\infty} G_{j}\left(\lambda^{-1 / 2} x+\xi\right)\right] & \psi\left(\lambda^{-1 / 2} x(b)+\xi\right) d x \\
& =\sum_{1}^{\infty} \int_{C_{0}[a . b]} G_{j}\left(\lambda^{-1 / 2} x+\xi\right) \psi\left(\lambda^{-1 / 2} x(b)+\xi\right) d x \\
& =\sum_{1}^{\infty}\left(I_{\lambda}^{\text {an }}\left(G_{j}\right) \psi\right)(\xi)=\left(L_{\lambda}(F) \psi\right)(\xi) .
\end{aligned}
$$

$\left(N(F)\left|\psi\left(\lambda^{-1 / 2} x(b)+\xi\right)\right|\right.$ serves as a dominating function.) So $I_{\lambda}^{\text {an }}(F)$ exists and equals $L_{\lambda}(F)$ and $\left\|I_{\lambda}^{\text {an }}(F)\right\| \leqq e^{|\lambda| / 2} N(F)$.

It remains to show $J_{q}^{\text {an }}(F)$ to be the strong operator limit of $I_{\lambda}^{\text {an }}(F)$ as $\lambda \rightarrow-i q$. Let

$$
L_{q}(F) \equiv \sum_{1}^{\infty} J_{q}^{\text {an }}\left(G_{j}\right)
$$

By (2.2), $L_{q}(F)$ is an operator and $\left\|L_{q}(F)\right\| \leqq e^{|q| / 2} N(F)$. Further the partial sums in (2.5) converge uniformly to $L_{q}(F)$ for $q$ in any bounded subset of $R-\{0\}$. Now fix $q \neq 0, \psi \in L_{2}(R)$ and let $\left\{\lambda_{n}\right\}$ be any sequence in $C^{+}$such that $\lambda_{n} \rightarrow-i q$. Using Lemma 2.1, uniform convergence, and the strong operator limit established in [3], we have

$$
\begin{aligned}
\lim _{n \rightarrow \infty} I_{\lambda_{n}}^{\mathrm{an}}(F) \psi & =\lim _{n \rightarrow \infty}\left[\lim _{M \rightarrow \infty} \sum_{1}^{M} I_{\lambda_{n}}^{\mathrm{an}}\left(G_{j}\right) \psi\right] \\
& =\lim _{M \rightarrow \infty}\left[\lim _{n \rightarrow \infty} \sum_{1}^{M} I_{\lambda_{n}}^{\mathrm{an}}\left(G_{j}\right) \psi\right] \\
& =\lim _{M \rightarrow \infty} \sum_{1}^{M} J_{q}^{\mathrm{an}}\left(G_{j}\right) \psi=L_{q}(F) \psi
\end{aligned}
$$

THEOREM 2.2. The sequential integral need not exist for $F \in A$. In fact, there exists a functional $F_{0}$ equivalent to 0 such that, for every $\lambda \in C^{+}$, $I_{?}^{\mathrm{seq}}\left(F_{0}\right)$ fails to exist. (Since $I_{\lambda}^{\mathrm{seq}}(F)$ clearly exists for the identically 0 
functional, this result shows that the sequential integral does not respect equivalence classes.)

Proof. We assume for convenience that $[a, b]=[0,1]$. Let

$$
\begin{aligned}
g\left(v_{1}, v_{2}\right) & =\exp \left(v_{1}^{4}\right), & & v_{2}=0, \\
& =0, & & \text { otherwise. }
\end{aligned}
$$

Let $\alpha_{j, 1}(t) \equiv 1$ and $\alpha_{j, 2}(t) \equiv 2^{1 / 2} \sin (2 j \pi t), j=1,2, \cdots$. Let

and

$$
g_{j}\left(v_{1}, v_{2}\right) \equiv 2^{-j} g\left(v_{1}, v_{2}\right)
$$

$$
\begin{aligned}
G_{j}(x) & \equiv g_{j}\left(\int_{0}^{1} \alpha_{j, 1}(t) d x(t), \int_{0}^{1} \alpha_{j, 2}(t) d x(t)\right) \\
& =2^{-j} g\left(x(1)-x(0), 2^{1 / 2} \int_{0}^{1} \sin (2 j \pi t) d x(t)\right) .
\end{aligned}
$$

Finally, let $F_{0}=\sum_{1}^{\infty} G_{j}$. Since $\left\|g_{j}\right\|=0$ for every $j, F_{0} \in A$ and, in fact, $F_{0}$ is equivalent to 0 .

To get the result, it suffices to exhibit a sequence $\left\{\sigma_{m}\right\}$ of partitions of $[0,1]$ such that $\left\|\sigma_{m}\right\| \rightarrow 0$ but, for each $m$ and each $\lambda \in C^{+}, I_{\lambda}^{\sigma_{m}}\left(F_{0}\right)$ is not even an operator. Let $\sigma_{m}=[0,1 / m, 2 / m, \cdots, 1]$. Let $\lambda \in C^{+}$. We will show that $I_{\lambda}^{\sigma_{m}}\left(F_{0}\right)$ is not an operator on $L_{2}(R)$ by giving an $L_{2}$-function $\psi_{0}$ such that $\left(I_{\lambda}^{\sigma_{m}}\left(F_{0}\right) \psi_{0}\right)(\xi)$ is not in $L_{2}(R)$. Take $\psi_{0}(u)=u^{-3 / 4} \chi_{[1, \infty)}(u)$. Let $\xi \in R$ with $\xi \neq 0$. By definition [2, p. 530, equation (4.7)],

$\left(I_{\lambda}^{\sigma_{m}}\left(F_{0}\right) \psi_{0}\right)(\xi)$

$$
\begin{aligned}
=\left(\frac{\lambda m}{2 \pi}\right)^{m / 2} \int_{-\infty}^{\infty}(m) \int_{-\infty}^{\infty} & \left\{\sum_{j=1}^{\infty} F_{j}\left[z\left(\sigma_{m}, \xi, v_{1}, \cdots, v_{m}, \cdot\right)\right]\right\} \\
\cdot & \psi_{0}\left(v_{m}\right) \exp \left(-\frac{\lambda m}{2} \sum_{i=1}^{m}\left(v_{i}-v_{i-1}\right)^{2}\right) d v_{1} \cdots d v_{m} \\
=\left(\frac{\lambda m}{2 \pi}\right)^{m / 2} \int_{-\infty}^{\infty}(m) \int_{-\infty}^{\infty}\{ & \left.\sum_{j=1}^{\infty} 2^{-j} g\left(v_{m}-\xi, 2^{1 / 2} \sum_{i=1}^{m}\left(v_{i}-v_{i-1}\right) \sin \left(\frac{2 j \pi i}{m}\right)\right)\right\} \\
\cdot & \psi_{0}\left(v_{m}\right) \exp \left(-\frac{\lambda m}{2} \sum_{i=1}^{m}\left(v_{i}-v_{i-1}\right)^{2}\right) d v_{1} \cdots d v_{m} .
\end{aligned}
$$

We will soon show that for certain values of $j$,

$$
2^{-j} g\left(v_{m}-\xi, 2^{1 / 2} \sum_{i=1}^{m}\left(v_{i}-v_{i-1}\right) \sin \left(\frac{2 j \pi i}{m}\right)\right)
$$

equals $2^{-j} \exp \left(v_{m}-\xi\right)^{4}$, and for all other values of $j,(2.6)$ is the 0 function 
in $\left(v_{1}, \cdots, v_{m}\right)$. Once this is shown, we will have

$$
\begin{aligned}
\left(I_{\lambda}^{\sigma m}\left(F_{0}\right) \psi_{0}\right)(\xi) & =\left(\frac{\lambda m}{2 \pi}\right)^{m / 2} \int_{-\infty}^{\infty}(m) \int_{-\infty}^{\infty} c_{0} \exp \left[\left(v_{m}-\xi\right)^{4}\right] \psi_{0}\left(v_{m}\right) \\
\cdot & \exp \left(-\frac{\lambda m}{2} \sum_{i=1}^{m}\left(v_{i}-v_{i-1}\right)^{2}\right) d v_{1} \cdots d v_{m} \\
= & c_{0}\left(\frac{\lambda}{2 \pi}\right)^{1 / 2} \int_{1}^{\infty} v_{m}^{-3 / 4} \exp \left[\left(v_{m}-\xi\right)^{4}-\frac{\lambda}{2}\left(v_{m}-\xi\right)^{2}\right] d v_{m}
\end{aligned}
$$

where $c_{0}$ is a positive constant. It is now clear that $\left(I_{\lambda}^{\sigma_{m}}\left(F_{0}\right) \psi_{0}\right)(\xi)$ is not an $L_{2}$-function of $\xi$.

It remains to verify the claim made for (2.6). Now

$$
\sum_{i=1}^{m}\left(v_{i}-v_{i-1}\right) \sin (2 j \pi i / m)
$$

is a linear combination $a_{0} \xi+a_{1} v_{1}+\cdots+a_{m-1} v_{m-1}$ of $\xi, v_{1}, \cdots, v_{m-1}$. If $a_{0}=a_{1}=\cdots=a_{m-1}=0$, then (2.6) equals $2^{-j} g\left(v_{m}-\xi, 0\right)=2^{-j} \exp \left(v_{m}-\xi\right)^{4}$. This can happen, for example, when $j=m, 2 m, \cdots$. On the other hand, if some one of $a_{0}, a_{1}, \cdots, a_{m-1}$ is not 0 , then (2.6) is 0 except for $\left\{\left(v_{1}, \cdots, v_{m}\right): a_{0} \xi+a_{1} v_{1}+\cdots+a_{m-1} v_{m-1}=0\right\}$; but this set has measure 0 in $R^{m}$.

\section{BIBLIOGRAPHY}

1. John A. Beekman and Ralph A. Kallman, Gaussian Markov expectations and related integral equations, Pacific J. Math. 37 (1971), 303-318.

2. R. H. Cameron and D. A. Storvick, An operator valued function space integral and a related integral equation, J. Math. Mech. 18 (1968), 517-552. MR 38 \#4643.

3. G. W. Johnson and D. L. Skoug, Operator-valued Feynman integrals of finitedimensional functionals, Pacific J. Math. 34.(1970), 415-425. MR 42 \#3625.

Department of Mathematics, University of Nebraska, Lincoln, Nebraska 68508 